\title{
Effect of ultrasound pretreatment on physicochemical properties of corn starch
}

\author{
Hao Zhang, Mei Li, Kun, Li, Chuanhe Zhu* \\ Key Laboratory of Food Processing Technology and Quality Control in Shandong Province, College \\ of Food Science and Engineering, Shandong Agricultural University, Taian 271018, China \\ *Corresponding author: Chuanhe Zhu, Email: chhzhu@sdau.edu.cn
}

Keywords: ultrasound pretreatment; corn starch; properties

Abstract. Effects of ultrasound pretreatment on physicochemical properties of corn starch were investigated. The results indicated that the transmittance and solubility of the starch increases by ultrasonic pretreatment. The starch pretreated with ultrasound for $30 \mathrm{~min}$ showed the maximum transmittance, which increased by $69.85 \%$. The starch pretreated by for 30 min shows the maximum solubility, and it is a $5.3 \%$ increase as compared with the native starch. Ultrasonic pretreatment was an effective modified method to alter the properties of corn starch.

\section{Introduction}

Starch is a widespread, cheap, degradable, abundantly available, pollution-free and renewable resource. But, corn starch has many short falls, i.e., insoluble in cold water, easy to dehydration, low emulsifying power and unstable in acid, which impede its uses. To overcome these disadvantages, some methods have been used to modify starch in attempting to increase its uses. [1].

Pretreatment with ultrasounds (sound waves at or above a frequency range of 15-20 kHz) is one of the physical modification methods of starch [2]. This process in contrast to chemical methods is considered to be environmentally friendly because it can reduce usage of chemicals, waste production and energy consumption [2]. According to some reports, ultrasounds cause physical degradation of granules with visible fissures and pores on the surface, the extent of granule damage is related to phenomenon of cavitation [4]. Starch granules are bombarded by bubbles of gases in the suspension medium before their collapse. Rapidly collapsing bubbles can cause arising of shear forces and micro-jets close to the surface of a granule which in turn results in its break-up. Moreover, solvent molecules may dissociate to form radicals which may induce polymer degradation [5]. Other factors affecting the ultra-sonication effect on starch granule include sonication power and frequency, amount of energy input, temperature and duration of the treatment, starch type and composition, starch concentration [6].

\section{Materials and methods}

\section{Materials}

Corn starch containing 22.45 $\pm 0.21 \%$ amylose was purchased from Shandong Xiangrui Pharmaceutical Co, Ltd (Taian, China). All other chemicals involved were of analytical grade. All solutions were prepared with distilled deionized water.

\section{Ultrasound pretreatment of corn starch}

Corn starch was suspended in a sufficient volume of distilled water to give different concentration $(10 \%, 20 \%, 30 \%, 40 \%, 50 \%, 60 \% \mathrm{w} / \mathrm{v})$. Prepared corn starch suspension was treated directly in the ultrasound reactor with the working volume of $2 \mathrm{~L}$ (Beijing Hongxianglong Biotechnology Co., Ltd.) at a frequency of $40 \mathrm{kHz}$. The reaction time (10, 20,30, 40, $50 \mathrm{~min})$, ultrasonic power $(240,300,360$, $420,480,540,600 \mathrm{~W})$ and reaction temperature $\left(30,40,50,60^{\circ} \mathrm{C}\right)$, are controlled during the reaction. At the bottom, the ultrasound reactor is equipped with an ultrasound transducer, ultrasound generator 
to produce high-frequency high-pressure, through the cable connection to the transducer, the sensor and vibration plate with high-frequency resonance. After treating with different ultrasound conditions, it was washed and filtered repeatedly with distilled water, and then starch slurry was dried to a moisture content of $10 \%$ in a hot air oven at $40{ }^{\circ} \mathrm{C}$ and milled to powder. Starch samples were stored in sealed plastic bags at room temperature before analysis.

\section{Solubility}

Solubility (S) was measured using the method reported by Adebowale, Afolabi (2005) [7]. Starch suspension $(2 \% \mathrm{w} / \mathrm{w})$ was prepared using a vortex mixer. $10 \mathrm{~mL}$ solution was transferred to a glass tube and heated by shaking at $85{ }^{\circ} \mathrm{C}$ for $30 \mathrm{~min}$, separately. Then, the samples were cooled to room temperature and centrifuged at $4000 \mathrm{~g}$ for $15 \mathrm{~min}$. The supernatant was decanted carefully. The residue obtained after drying the supernatant represented the amount of starch dissolved in water. Solubility was calculated per $100 \mathrm{~g}$ of starch on dry weight basis. The supernatant was poured into a weighted aluminum pan before drying to a constant weight (W1) at $105^{\circ} \mathrm{C}$.

$$
\mathrm{S}(\%)=\mathrm{W} 1 / \mathrm{W} 0 \times 100 \%
$$

\section{Transparency}

Transparency was measured according to method described by Li Mei [4]. Starch (2 g/100 g dry basis) aqueous mixture was heated and stirred in water bath for $15 \mathrm{~min}$ at $95^{\circ} \mathrm{C}$. Samples were cooled at $25{ }^{\circ} \mathrm{C}$ and percent transmittance was measured at $620 \mathrm{~nm}$ against water blank using a spectrophotometer (UV-754N, Shanghai APLSH instrument Co., Ltd, China).

\section{Statistical analyses}

All runs were conducted in triplicate, and the results were expressed as mean \pm SD of triplicate treatments unless stated otherwise. Analysis of variance (ANOVA; $p<0.05$ ) and Duncan's multiple range tests were used to evaluate the differences between the different groups. All analyses including calculations were conducted with the aid of the statistical software SPSS 21.0, and the graphs were created in Origin 8.5.

\section{Results and discussion}

\section{Effect of different ultrasound pretreatment time on the solubility of corn starch}
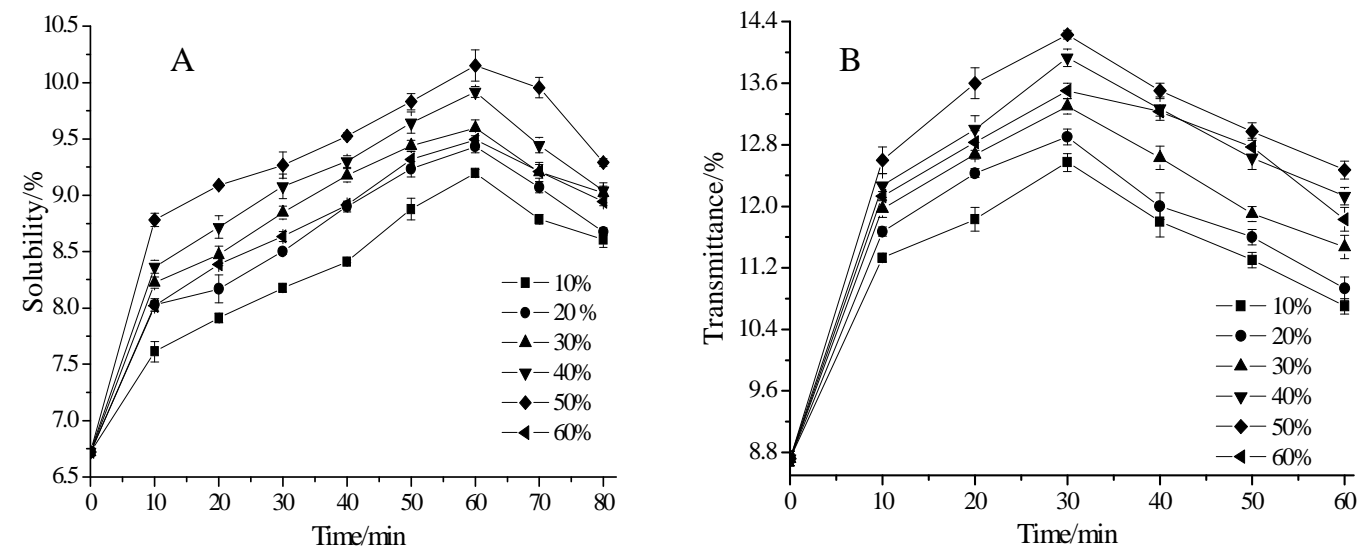

Fig. 1 Solubility and transparency of corn starches under different ultrasound pretreatment time. A Solubility; B Transparency

The Solubility of corn starches solutions pretreated with different ultrasound time is displayed in Fig. 1. As can be seen from Fig. 1A, the solubility of cornstarch after ultrasonic pretreatment is higher 
than that of native starch, and increases with the increase of ultrasonic time first and then decreases. This is because with the extension of ultrasound time, ultrasound damage starch crystallization zone, the molecular bond within the particle fracture. Both the surface and the interior of the particles are eroded, and the association between starch and water molecules increases, resulting in an increase in the solubility of the starch [8].

\section{Effect of different ultrasound pretreatment time on the transparency of corn starch}

As can be seen from Fig. 1B, the transparency of sonicated starch is greatly increased compared with the native starch, and the transparency of the starch reaches the maximum at $50 \%$ treating for 30 min, then gradually decreases. Ultrasound may damage the starch crystallization zone in a relatively short period of time, and the surface and internal structure of the particles are destroyed, resulting in an increase in the solubility of the starch. The associativity between starch and water molecules increases and the association between molecules decreases. Starch granules tend to swell, there by weakening the refraction and reflection of light, resulting in increased transparency of the starch paste. And because with the extension of ultrasound time will cause reunion of starch particles, inhibit particle expansion, resulting in a relative decline in transparency. Therefore, the transparency of starch after ultrasonic treatment showed a trend of first increase and then decrease with the extension of treatment time [9].

\section{Effect of different ultrasound pretreatment power on the solubility of corn starch}
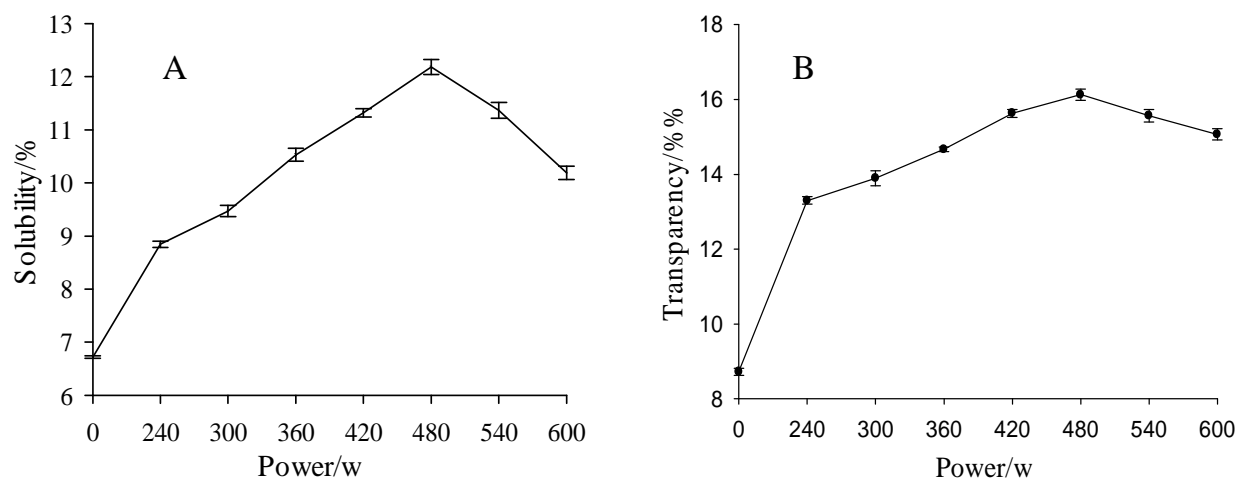

Fig. 2 Solubility and transparency of corn starches under different ultrasound pretreatment power. A

Solubility; B Transparency

As the power of ultrasound increased, the solubility of the pores progressively augmented until 480W (Fig. 2A); at higher power level no further solubility increase was observed. This is because the solubility of starch is mainly determined by the amount of amylose liberated from the starch granules. With the increase of ultrasonic power, the molecular bonds in the starch granules break, making the granules become loose and the starch molecules are easily dissolved from the granules, so that the solubility of the starch is increased [10].

\section{Effect of different ultrasound pretreatment power on the transparency of corn starch}

Pretreated corn starches showed a noticeable increase in transparency $(\mathrm{p}<0.05)$. The transparency of corn starches increased with increasing ultrasound power $(<480 \mathrm{w})$ (Fig.2B). The reason for this phenomenon may be that the surface and internal structure of the particles are damaged after ultrasonication, the solubility of the starch is increased, and the particles are more easily swelled, thereby weakening the refraction and reflection of light and improving the transparency of the starch paste. Ultrasonic power continues to increase will cause the destruction of particles is too large, re-agglomeration of starch particles, inhibition of particle expansion, resulting in a relative decline in transparency [5]. 


\section{Effect of different ultrasound pretreatment temperature on the solubility of corn starch}
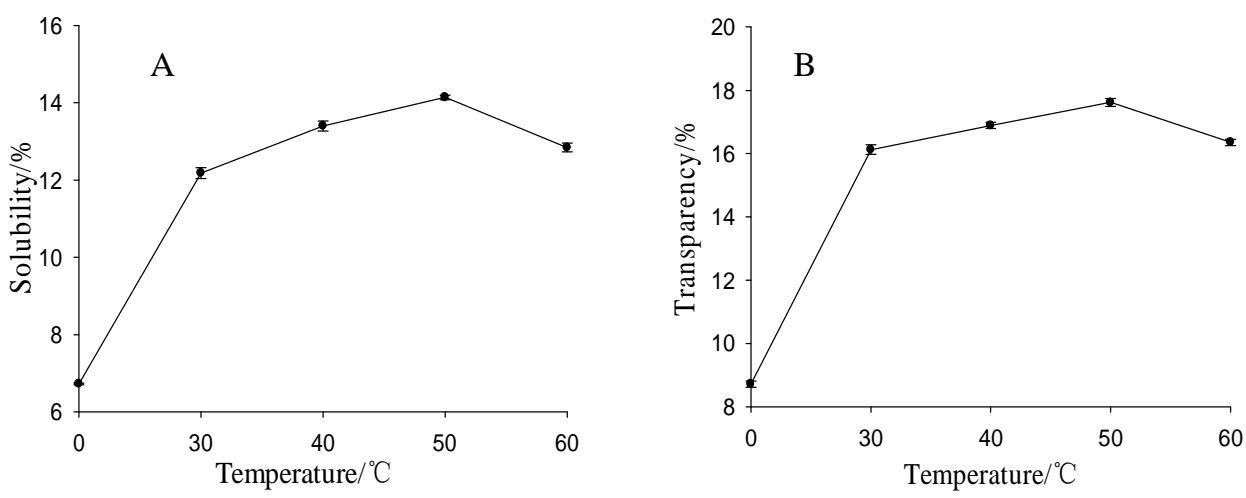

Fig. 3 Solubility and transparency of corn starches under different ultrasound pretreatment temperature. A

Solubility; B Transparency

Solubility of corn starches was promoted by the increasing ultrasound temperature until reaching the optimum level at $50{ }^{\circ} \mathrm{C}$ (Fig.3A). However, solubility decreased when temperature was further raised. Ultrasound breaks the molecular bonds in the particles, loosening the structure of the particles, and freeing the amylose to dissolve in water to increase the solubility of the starch. As the ultrasonic temperature increases, the heat released increases, so that the degree of starch gelatinization increases, affecting the ultrasound effect. Therefore, the solubility of starch decreases after ultrasonication at $50{ }^{\circ} \mathrm{C}[11]$.

\section{Effect of different ultrasound pretreatment temperature on the transparency of corn starch}

As can be seen from Fig. 3B, the transparency of the starch after ultrasound is greatly improved compared to the native starch, and the transparency of the starch paste at the ultrasonic treatment at $50{ }^{\circ} \mathrm{C}$ reaches a maximum and then gradually decreases. It may be that the ultrasonic energy destroys the crystalline area of the starch within a certain temperature range, and the surface and internal structure of the particles are destroyed, resulting in increased solubility of the starch. Starch granules easier to expand, thereby reducing the light refraction and reflection, so that the transparency of starch paste increased. The ultrasonic temperature continues to increase, the heat released increases, the degree of starch gelatinization increases, affecting the ultrasound effect. It will also cause the re-agglomeration of starch granules, inhibiting the swelling of the granules, leading to a relative decrease in transparency [12].

\section{Conclusions}

Ultrasonic technology was applied to treat corn starch. The optimal ultrasonic conditions were as follows: concentration $50 \%$, ultrasonic time $30 \mathrm{~min}$, ultrasonic power $480 \mathrm{w}$, ultrasonic temperature $50{ }^{\circ} \mathrm{C}$. Under this condition, the verification experiment was carried out. The solubility of starch in ultrasonic was $14.22 \%$ and the transparency was $17.71 \%$. Compared with the physicochemical properties before and after ultrasound, its transparency and solubility have increased.

\section{Acknowledgements}

This work was financially supported by the Funds of Shandong "Double Tops" Program (SYT2017XTTD04), Shandong province science and technology plan projects (J14LF11). 


\section{References}

[1] B. Kaur, F. Ariffin, R. Bhat and A. Karim: Progress in starch modification in the last decade, Food Hydrocoll. 26.2: 398-404 (2012).

[2] D. Wang, X. Ma, L. Yan, T. Chantapakul, W. Wang, T. Ding, X. Ye and D. Liu: Ultrasound assisted enzymatic hydrolysis of starch catalyzed by glucoamylase: Investigation on starch properties and degradation kinetics. Carbohydr. Polym. 175. 47-54 (2017).

[3] P. Tomasik and M. F. Zaranyika: Nonconventional methods of modification of starch, Adv. Carbohydr. Chem. Biochem. 51. 243-320 (1995).

[4] M. Li, J. X. Li and C. H. Zhu: Effect of ultrasound pretreatment on enzymolysis and physicochemical properties of corn starch. International Journal of Biological Macromolecules. 111. 848-856 (2018).

[5] Y.Y.J. Zuo, P. Hébraud, Y. Hemar and M. Ashokkumar: Quantification of high-power ultrasound induced damage on potato starch granules using light microscopy. Ultrason. Sonochem. 19. 421-426 (2012).

[6] W. Bai, P. Hébraud, M. Ashokkumar and Y. Hemar: Investigation on the pitting of potato starch granules during high frequency ultrasound treatment, Ultrason. Sonochem. 35. 547-555 (2016).

[7] K. O. Adebowale, T. A. Afolabi, and B. I. Olu-Owolabi: Hydrothermal treatments of finger millet (Eleusine coracana) starch. Food Hydrocolloids. 19. 974-983 (2005).

[8] L. H Lan, D. X. Li and A. M. Chen: Effects of Ultrasound on Crystallization of Cassava Starch. Journal of Anhui Agri. Sci. 40.17: 9192-9195 (2012), p9208.

[9] L. Lin and J. B. Li: Effect of Ultrasound on the Properties of Potato Starch Pastes. Journal of South China University of Technology (Natural Science Edition). 36.11: 63-67 (2012).

[10] S. Tsubaki, H. Iida, M, Sakamoto and J Azuma: Microwave Heating of Tea Residue Yields Polysaccharides, Polyphenols, and Plant Biopolyester. J Agric Food Chem. 56.23: 11293-9 (2008).

[11] J. Anetrezek, H. Zoran, S. Drago, B. Jurislav and B Mladen: Ultrasound effect on physical properties of corn starch. Carbohydrate Polymers. 79.1: 91-100 (2010).

[12] Y. Q. Zhao, X. W. He and Q. Huang: Crystallinity properties of starch granule and non-crystallization methods for it. Modern Chemical Industry. 27.11: 67-69 (2007). 